\title{
Las operaciones básicas y el método heurístico de Pólya como pretexto para fortalecer la competencia matemática resolución de problemas
}

\section{Basic operations and the heuristic method of Polya as a pretext for strengthening mathematical competence in problem solving}

\author{
GUALDRÓN, Elgar ${ }^{1}$ \\ PINZÓN, LuZ ${ }^{2}$ \\ ÁVILA, Adriana ${ }^{3}$
}

\begin{abstract}
Resumen
El presente artículo deriva de un trabajo de investigación cuyo propósito fue fortalecer la competencia resolución de problemas en estudiantes de cuarto en una institución educativa haciendo uso en contexto de operaciones matemáticas básicas, el método heurístico de Pólya y la resolución misma de problemas. El enfoque fue cualitativo con un diseño de investigación acción. Las acciones implementadas lograron contextualizar la matemática, motivar a los estudiantes en la búsqueda de estrategias de resolución a los problemas y mejorar su desempeño.
\end{abstract}

Palabras clave: competencia matemática, resolución de problemas, pensamiento numérico

\begin{abstract}
This article come from a research work it purpose was to strengthen problem solving in fourth-year students in an educational institution making use in the context of basic mathematical operations, the heuristic method of Pólya and problem solving. The approach was qualitative with an action research design. The implemented actions managed to contextualize mathematics, motivate students in the search for problem solving strategies and improve their performance.

Key words: mathematical competence, problem solving, number thinking
\end{abstract}

\section{Introducción}

Los problemas matemáticos transforman las prácticas pedagógicas, implican un modo de pensar, razonar, explorar y crear, que se utilizan para resolver toda clase de problemas en las ciencias, la investigación científica, el gobierno, la industria y otros campos altamente competitivos (Camero, Martínez \& Pérez, 2016; Phonapichat, Wongwanich \& Sujiva, 2014). Así mismo, el verdadero sentido de la matemática se da cuando se aplica en contexto para resolver situaciones de la vida diaria que implican para el estudiante el desarrollo de procesos del pensamiento matemático (Sánchez \& Fernández, 2003; Li, Zhoua, Huanga, Tuc, Gaod, Yanga \& Lia, 2020).

\footnotetext{
${ }^{1}$ Doctor en Didáctica de las Matemáticas. Docente de la Universidad de Pamplona (Colombia), Departamento de Matemáticas. Director del grupo de Investigación EDUMATEST_Universidad de Pamplona. egualdron@unipamplona.edu.co

2 Estudiante Maestría en Educación. Universidad Autónoma de Bucaramanga. Ipinzon888@unab.edu.co

${ }^{3}$ Doctora en Educación. Docente de la Facultad de Ciencias Sociales, Humanidades y Artes. Universidad Autónoma de Bucaramanga. aavila2@unab.edu.co
} 
En muchas ocasiones, en el ámbito escolar los docentes se dedican a la matemática formal, abstracta y operacional, dejando un espacio casi nulo para la resolución de problemas, y menos aún para su contextualización con la realidad. Es así como los diferentes gobiernos han creado políticas educativas que buscan fortalecer estos procesos y brindar a la población una educación de calidad (Ministerio de Educación Nacional de Colombia, MEN, 2006), ejemplo de ello son los Estándares Básicos de Competencias, los Lineamientos Curriculares y los Derechos Básicos del Aprendizaje, orientados a mejorar el nivel de desempeño de los estudiantes, la competitividad y el rendimiento de los profesionales en el ámbito laboral (Gómez \& Velasco, 2017). El método heurístico de George Pólya para resolución de problemas ha sido un importante referente para diversos estudios (López-Leyton, Aldana \& Erazo, 2019; Valbuena, Muñiz \& Berrio, 2020), que reportan resultados favorables en el desarrollo de esta competencia en estudiantes.

En las pruebas Saber $3^{\circ}$ y $5^{\circ}$ realizadas en Colombia durante el año 2017 se observó la dificultad que presentan los estudiantes en la competencia resolución de problemas y razonamiento matemático (Instituto Colombiano para la Evaluación de la Educación, ICFES, 2018); los resultados evidencian que hay una brecha entre lo alcanzado por los estudiantes y lo establecido por el Ministerio de Educación de Colombia, respecto a cómo la resolución de problemas debe ser la base de los procesos de la planeación curricular del área de matemáticas, donde prevalezca la contextualización de situaciones que den sentido a la disciplina y dejen ver la viabilidad y aplicación de procesos matemáticos en el diario vivir (MEN, 2006). Además, siguiendo a Sánchez \& Fernández (2003), aprender matemáticas requiere que los procesos de enseñanza estén mediados por métodos y técnicas que promuevan en los estudiantes el deseo de analizar, descubrir y relacionar patrones, que les permitan aplicarlos en los diferentes procesos, y con ello generar nuevo conocimiento que modifique los constructos ya preestablecidos.

Considerando las ideas anteriores, surgió la necesidad de plantear un trabajo de investigación orientado a fortalecer la competencia resolución de problemas, en los estudiantes de grado cuarto de una institución pública rural del departamento de Santander-Colombia, en atención a que su desempeño denotaba dificultades, y así mismo generar condiciones para mejorar las prácticas pedagógicas de los docentes de matemáticas.

En el presente artículo se exponen los resultados obtenidos en dicho trabajo, luego de una experiencia de diseño, desarrollo e implementación de diferentes acciones pedagógicas que finalmente se concretaron en la construcción de una unidad didáctica que, mediante actividades secuenciales y motivadoras, teniendo en cuenta las operaciones básicas como contexto y el método heurístico de Pólya, se centró en fortalecer la competencia resolución de problemas.

La unidad didáctica desarrollada y aplicada considera dos fases de trabajo, una práctica que incluye el objetivo y las secciones: "es hora de practicar", "es hora de crear" y "cuánto he aprendido" y, una segunda fase, de fortalecimiento de procesos con el desarrollo de una ficha que incluye cálculo matemático, resolución y redacción de problemas. La estrategia didáctica en resolución de problemas permitió al docente contextualizar los saberes matemáticos de los estudiantes y mediante la aplicación de actividades pudo observar su avance, teniendo en cuenta de manera objetiva la aplicación de saberes y la implementación de metodologías basadas en conjeturas e hipótesis, donde los docentes encontraron estrategias de solución y resultados verificables, lo que constituyó la construcción de nuevo conocimiento. En el desarrollo, se aplicó la metodología investigación acción, propia del enfoque cualitativo. Se partió de una prueba diagnóstica, luego se diseñó una unidad didáctica base, que se fue implementando y de manera paralela enriqueciendo y ajustando de acuerdo con la experiencia y su aporte al mejoramiento de resolución de problemas; finalmente, se aplicó una prueba que mostró los avances alcanzados por los estudiantes en el fortalecimiento de la competencia mencionada y el pensamiento numérico 


\subsection{Referentes teóricos}

Problema matemático: se define problema matemático como un "enunciado" que incluye datos relevantes y requiere la aplicación de un plan para ser solucionado; debe poseer un lenguaje matemático claro donde se utilicen palabras y símbolos; y, además, emplee variables alfabéticas y numéricas, para obtener una respuesta acertada (Pólya, 1965). El problema debe estar formulado y adaptado al nivel de los conocimientos de los estudiantes (Godino, Batanero \& Font, 2003).

Método heurístico resolución de problemas: los estudios de Pólya (1965) sugieren que los métodos heurísticos son estrategias y reglas generales, utilizadas para solucionar problemas, que incluyen operaciones mentales basadas en experiencias previas con problemas similares, e indican el camino a seguir para alcanzar una solución.

Así mismo, Pólya (1965) expone ideas sobre cómo ayudar a los alumnos a pensar por sí mismos e indica cuatro pasos: "entender o interpretar un problema, concebir un plan, ejecutar el plan y visión retrospectiva o examinar la solución" (p.17). Estos pasos se asocian a una lista de preguntas, donde el docente: pruebe la curiosidad del estudiante, despierte el gusto por el pensamiento, el descubrimiento y el deseo de implementar estrategias de solución, que se refleja en el triunfo y determina una afición por el trabajo intelectual, donde la matemática cobra sentido para él.

Competencias matemáticas: definidas por las pruebas externas PISA (citado en Organización para la Cooperación y Desarrollo Económicos, OCDE, 2017) como: "la capacidad del individuo para formular, emplear e interpretar las matemáticas en distintos contextos" (p. 64). Esto requiere: analizar, comprender y argumentar una determinada situación matemática cotidiana, que genera una inquietud, permite implementar habilidades y procesos de razonamiento y necesita ser resuelta (Mazzilli, Hernández \& De La Hoz, 2016).

Unidad didáctica: Según García-Aretio (2009), una unidad didáctica es:

Un conjunto organizado y secuencial de los elementos básicos que conforman el proceso de enseñanzaaprendizaje (motivación, relaciones con otros conocimientos, objetivos, contenidos, métodos y estrategias, actividades y evaluación) con sentido propio, unitario y completo que permite a los estudiantes, tras su estudio, apreciar el resultado de su trabajo. (p. 1)

Una unidad didáctica, para la resolución de problemas, debe propiciar que los estudiantes integren los conocimientos previos con los nuevos de manera organizada, que incluya cálculo matemático, análisis, comprensión y resolución de problemas de forma secuencial. Además, debería estar conformada por actividades que se desarrollen en equipo y de forma individual, donde prevalezca la implementación del método heurístico de Pólya, mediante situaciones que lleven a los estudiantes a cuestionarse, proponer y deducir estrategias para llegar a respuestas precisas y verificables. Santos-Trigo (2008) afirma que "es importante promover un currículum en términos de secuencias de problemas, donde se reflejen los aspectos inherentes que transforman las asignaturas tradicionales en líneas de pensamiento numérico, algebraico, numérico y estadístico" (p. 21). En este sentido, el éxito del proceso enseñanza y aprendizaje depende de guías didácticas bien elaboradas y motivadoras, que promuevan en los estudiantes el gusto e interés por desarrollar las actividades matemáticas, que los incentive a adquirir y aplicar conocimientos, analizar e interpretar eventos, en la construcción del saber, del saber hacer y del saber ser (García-Aretio, 2014).

En síntesis, es necesario implementar actividades secuenciales que involucren situaciones problema, donde se contextualicen los conocimientos matemáticos y los estudiantes puedan explorar y plantear preguntas. Además de reflexionar sobre los pasos a seguir para resolverlas, tantas veces como sea necesario, promoviendo el desarrollo de habilidades y las competencias específicas del área. 


\section{Metodología}

La metodología se basó en la investigación-acción, planteada bajo el enfoque cualitativo, donde se pretendió, mediante la observación participante, describir de manera objetiva las actividades que se realizaron en el aula y, a la vez, reflexionar y construir conocimientos que permitieran fortalecer la competencia resolución de problemas, a través de una unidad didáctica centrada en la resolución de problemas matemáticos, para los estudiantes del grado cuarto.

El proceso metodológico de la investigación se fundamentó en la observación participativa y el análisis e interpretación de la información, lo cual permitió al docente investigador estar inmerso en la realidad investigada, tal como es y examinarla de forma natural (Marín, 2018). En este sentido, se hizo seguimiento a las actitudes, acciones e interacciones de los estudiantes en el desarrollo de las diferentes actividades, en las cuales prevaleció el diseño de estrategias, acciones y actividades que aportasen soluciones transformadoras a situaciones surgidas en el aula.

El diseño de la investigación se llevó a cabo en cuatro etapas (Sandín, 2003), a saber: diagnóstico, diseño, implementación y evaluación, y reflexión. A continuación, se describe cada etapa:

El diagnóstico: en esta etapa se diseñó y aplicó una prueba diagnóstica-cognitiva, construida en base a los conocimientos previos de cálculo matemático e incluyó operaciones básicas como: adición, sustracción, multiplicación y división, además de cuatro problemas propuestos para ser resueltos. La prueba buscaba determinar los procesos empleados por los estudiantes y el nivel de desempeño al resolver problemas matemáticos.

Diseño de la estrategia didáctica: en esta etapa se diseñó la unidad didáctica, conformada por actividades en las cuales se debe aplicar el método Pólya en resolución de problemas.

La unidad didáctica se desarrolló en dos fases: una actividad práctica con manejo de material manipulativo, en la cual se incluyeron las etiquetas "es hora de practicar", "es hora de crear", y "cuánto he aprendido"; trabajo que se realizó en diferentes contextos de la sede educativa, como: el aula de clase, aula de informática, cancha deportiva y alrededores. La otra fase implicó el desarrollo de una ficha de trabajo, que incluyó cálculo matemático de: adición, sustracción, multiplicación o división y planteamiento de problemas contextualizados.

Implementación y evaluación de la estrategia: en esta etapa se implementó la unidad didáctica, cuyo objetivo estaba direccionado a fortalecer la competencia resolución de problemas. Para ello, se diseñaron actividades que incluían problemas matemáticos que estimularon en los estudiantes la forma de pensar, analizar y encontrar una respuesta que cumpliera las condiciones dadas. El proceso fue mediado de forma bidireccional por el docente, quien, mediante preguntas, les permitió a los estudiantes buscar estrategias de resolución para llegar a respuestas verídicas (Santos-Trigo, 2008).

Por consiguiente, se planificaron y estructuraron actividades basadas en contenidos secuenciales, que incluían ideas clave y condujeron a los estudiantes a buscar una estrategia de solución para los problemas propuestos. Esto les permitió implementar de forma ordenada el método heurístico en resolución de problemas de Pólya, donde prevalecieron los conocimientos previos en la construcción del nuevo saber.

Para evaluar la estrategia, se parte de los aportes de Villalobos (2008), quien indica que la evaluación debe ser oportuna, acertada y variada, que permita comprobar las diferentes estrategias que emplean los estudiantes a la hora de aplicar los conocimientos adquiridos en la resolución de problemas. Por ello, se diseñó una prueba diagnóstica final, estructurada y conformada por ocho problemas, que comprendían: un enunciado con datos relevantes en forma de pregunta o afirmación incompleta y cuatro opciones de respuesta, una opción correcta 
y las demás incorrectas. Cada problema requería de un plan para ser solucionado; además, incluía redactar un problema con un enunciado completo y una o más variables.

Reflexión de la estrategia: en esta etapa se analizaron los datos obtenidos en las pruebas inicial y final, así como los datos recogidos en la observación participante, realizada a lo largo de la implementación de la estrategia didáctica en resolución de problemas.

\subsection{Categorías de análisis}

La muestra objeto de estudio estuvo constituida por 24 estudiantes del grado cuarto de la básica primaria. Las edades de los estudiantes se encontraban entre 9 y 11 años: 10 niñas y 14 niños, de los estratos socioeconómicos 1,2 y 3.

\section{Cuadro 1}

Categorías, subcategorías y técnicas e instrumentos de recolección de datos

\begin{tabular}{|c|c|c|c|c|}
\hline Categoría de análisis & $\begin{array}{l}\text { Código } \\
\text { categoría }\end{array}$ & $\begin{array}{c}\text { Subcategoría de } \\
\text { análisis }\end{array}$ & $\begin{array}{c}\text { Código } \\
\text { subcategoría }\end{array}$ & $\begin{array}{l}\text { Técnicas e instrumentos de } \\
\text { recolección de datos }\end{array}$ \\
\hline \multirow{2}{*}{$\begin{array}{l}\text { Relación entre reconocer y } \\
\text { aplicar el razonamiento } \\
\text { matemático al interpretar } \\
\text { información para resolver } \\
\text { problemas }\end{array}$} & \multirow[t]{2}{*}{ (C.1 Relación) } & $\begin{array}{l}\text { Realiza operaciones } \\
\text { matemáticas básicas }\end{array}$ & (C.1 Relación 1) & \multirow{2}{*}{$\begin{array}{l}\text { Observación participante } \\
\text { Prueba diagnóstica } \\
\text { Fichas de la unidad didáctica }\end{array}$} \\
\hline & & $\begin{array}{l}\text { Utiliza estrategias para } \\
\text { resolver problemas }\end{array}$ & (C.1 Relación 2) & \\
\hline \multirow{5}{*}{$\begin{array}{l}\text { Resolución de problemas que } \\
\text { integren razonamiento } \\
\text { matemático, modelización y } \\
\text { demostraciones para llegar a } \\
\text { deducciones }\end{array}$} & \multirow[t]{5}{*}{$\begin{array}{l}\text { (C.2 } \\
\text { Resolución) }\end{array}$} & $\begin{array}{l}\text { Identifica los datos, si } \\
\text { son suficientes o hay } \\
\text { información extraña y } \\
\text { las variables }\end{array}$ & (C.2 Resolución 1) & \multirow[t]{5}{*}{$\begin{array}{l}\text { Observación participante } \\
\text { Fichas de la unidad didáctica }\end{array}$} \\
\hline & & $\begin{array}{l}\text { Analiza cómo puede } \\
\text { resolver el problema, } \\
\text { concebir la idea de un } \\
\text { plan y buscar una } \\
\text { estrategia de resolución }\end{array}$ & (C.2 Resolución 2) & \\
\hline & & $\begin{array}{l}\text { Resuelve el problema } \\
\text { planteado }\end{array}$ & (C.2 Resolución 3) & \\
\hline & & $\begin{array}{l}\text { Comprueba la } \\
\text { respuesta por medio de } \\
\text { operaciones } \\
\text { matemáticas }\end{array}$ & (C.2 Resolución 4) & \\
\hline & & $\begin{array}{ll}\text { Crear } & \text { problemas } \\
\text { similares } & \\
\end{array}$ & (C.2 Resolución 5) & \\
\hline $\begin{array}{l}\text { Impacto de la implementación de } \\
\text { una unidad didáctica, donde se } \\
\text { integran procesos cognitivos para } \\
\text { fortalecer la competencia } \\
\text { resolución de problemas }\end{array}$ & (C.3 Impacto) & $\begin{array}{l}\text { Aplica estrategias para } \\
\text { resolver problemas }\end{array}$ & (C.3 Impacto 1) & $\begin{array}{l}\text { Observación participante } \\
\text { Fichas unidad didáctica }\end{array}$ \\
\hline $\begin{array}{l}\text { Resultados obtenidos al } \\
\text { implementar una unidad } \\
\text { didáctica en resolución de } \\
\text { problemas }\end{array}$ & $\begin{array}{l}\text { (C.4 } \\
\text { Resultados). }\end{array}$ & $\begin{array}{l}\text { Fortalecimiento de la } \\
\text { competencia resolución } \\
\text { de problemas mediante } \\
\text { el método George Pólya }\end{array}$ & (C.4 Resultados 1) & Prueba final \\
\hline
\end{tabular}

Fuente: elaboración propia

Las categorías de análisis se diseñaron teniendo en cuenta los referentes de calidad tales como: Estándares Básicos de Competencias (MEN, 2006), Lineamientos Curriculares de Matemáticas (MEN, 1998) y Derechos Básicos de Aprendizaje (MEN, 2016), con el fin de analizar y reflexionar la información recolectada en la prueba diagnóstica, la observación directa, las producciones realizadas por los estudiantes y la prueba final. A manera 
de resumen, el cuadro 1 muestra las categorías de análisis, los códigos correspondientes usados en el estudio, la subcategoría y el código correspondiente y, al final, el instrumento donde previsiblemente aparece la categoría o subcategoría.

\section{Resultados}

Sobre la prueba diagnóstica: una vez realizada y analizada la prueba diagnóstica, se observó que:

i) Los estudiantes presentaban dificultad al realizar cálculos matemáticos de sustracciones con cantidades que incluyen ceros en sus cifras, ya que no tenían claro los procedimientos de agrupación y desagrupación de cantidades, lo cual se convierte en impedimento para solucionar problemas.

ii) En los cálculos matemáticos de multiplicación y división se observó que los estudiantes presentaban dificultad al resolver estas operaciones ya que no tenían claro el proceso de resolución, lo cual les impedía aplicarlas de forma precisa en la solución de problemas cotidianos.

iii) En la resolución de problemas que involucran las operaciones de adición, sustracción, multiplicación y división, los estudiantes presentaban gran dificultad, parecían aplicar estas operaciones al azar, sin tener en cuenta los datos ni las variables expuestas en los enunciados. Es decir, no tenían clara la relación entre el reconocer y aplicar razonamiento matemático para interpretar la información y deducir la operación matemática, que les permitiera resolver los problemas propuestos.

El nivel de desempeño de los estudiantes, en la prueba diagnóstica, al resolver problemas matemáticos, se valoró con una escala de tres niveles (bajo, básico y alto) en la que bajo significa que el estudiante no resolvió ningún problema o solo resolvió uno o dos; básico alude a que se resolvieron tres ejercicios; alto representa que se resolvieron los cuatro problemas propuestos. El desempeño de los estudiantes se muestra en el cuadro 2.

Cuadro 2

Nivel de desempeño de los estudiantes en la prueba diagnóstica

\begin{tabular}{|c|c|c|}
\hline Problemas & Valoración & \# de estudiantes \\
$0-2$ & Bajo & 17 \\
3 & Básico & 7 \\
4 & Alto & 0 \\
\hline
\end{tabular}

Fuente: elaboración propia

Se evidencia como el $71 \%$ de los estudiantes se ubican en el nivel de desempeño bajo y el 29 \% en el básico, ninguno alcanzó un desempeño alto.

A continuación, en la figura 1, se presenta un ejemplo, de cómo un estudiante resolvió la prueba diagnóstica y las dificultades allí identificadas. 
Figura 1

Ejemplo de resolución

de la prueba diagnóstica

1. Realiza las siguientes operaciones
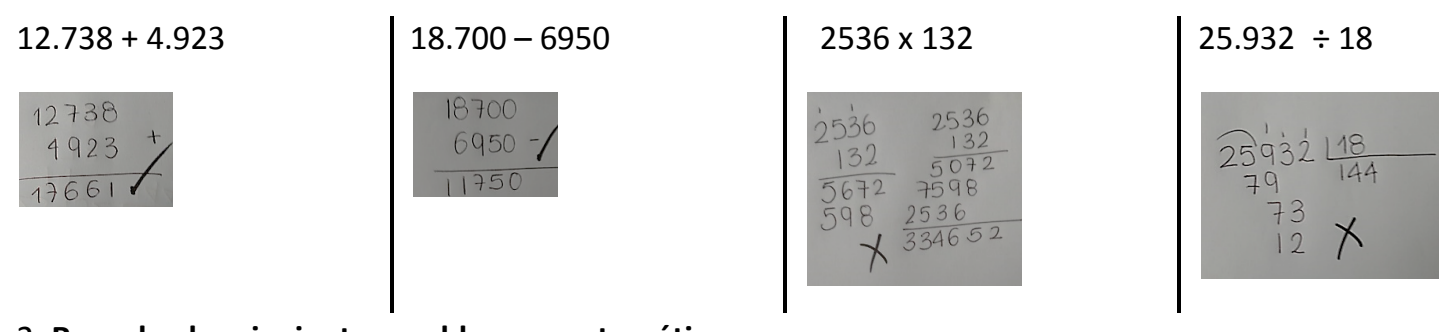

2. Resuelve los siguientes problemas matemáticos

En un tanque se depositan 1.200 litros de agua el lunes, 1.754 litros de agua el martes y 1.846 litros de agua el miércoles.

¿Cuántos litros de agua hay en el tanque?
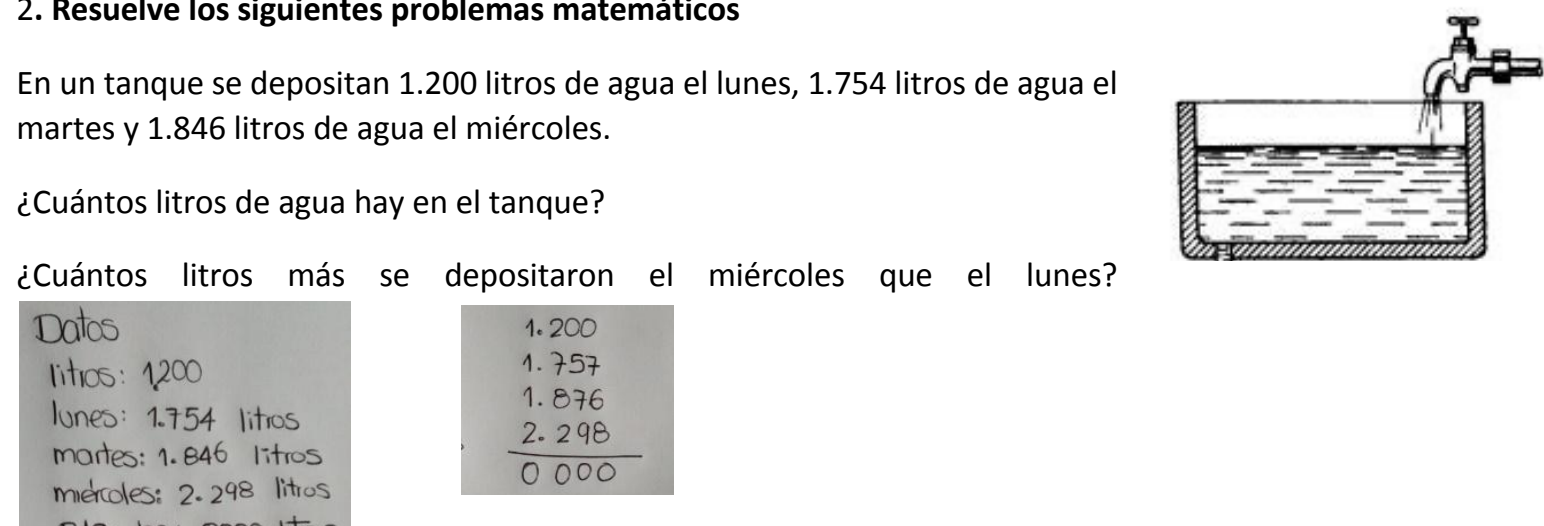

Fuente: Prueba diagnóstica del estudiante \# 1

La producción escrita de este estudiante evidencia su logro en las operaciones de adición y sustracción, pero la dificultad en el proceso que conduce al desarrollo de la multiplicación y la división. El estudiante evidencia la clásica estructura de resolución de problemas: determinación de datos, operaciones y respuesta; sin embargo, la organización de los datos no corresponde con la situación, en consecuencia, la operación que planteó contiene cifras que no son pertinentes, el resultado obtenido no es coherente y pareciera ser un intento por responder solo la primera pregunta; no se evidenció esfuerzo alguno por responder la segunda parte del problema.

Sobre la implementación de la unidad didáctica: una vez recolectada la información en los diarios de campo, se realizó un análisis descriptivo de las producciones de los estudiantes, teniendo en cuenta: el objetivo, las categorías y subcategorías de análisis, al igual que el trabajo desempeñado por cada uno, durante el desarrollo de las actividades propuesta en la unidad didáctica, la cual como se ha mencionado, tiene dos fases:

Fase 1: actividad práctica y de manejo de material manipulativo, en la cual se incluye el objetivo, por ejemplo: comprender y diseñar un plan para resolver problemas que involucren multiplicaciones de números naturales. Esta fase está organizada en tres secciones: la primera llamada "es hora de practicar" donde los estudiantes manipulaban materiales concretos a manera de juego y de manera libre, dentro y fuera del aula con el fin de acercarse a las operaciones matemáticas básicas, así como al análisis de problemas en contexto; la segunda, "es hora de crear" donde los estudiantes organizados en grupos crean situaciones problemas para retar a sus demás compañeros; la tercera, "cuánto he aprendido" los estudiantes resuelven una ficha en la cual ponen a prueba lo trabajando en la secciones anteriores.

Fase 2: desarrollo de una nueva ficha de trabajo, que incluye: cálculo matemático de adición, sustracción, multiplicación o división, y planteamiento de problemas contextualizados para ser resueltos. 
Para el desarrollo de las fichas de trabajo los estudiantes implementaron el paso a paso del método heurístico de Pólya guiado por preguntas que complementan su aplicación:

i) Comprender el problema: correcta interpretación del enunciado verbal, es entender lo que se dice, por medio de preguntas como: ¿Cuáles son los datos? ¿Cuál es la incógnita? ¿Cuál es la condición? ¿Sabes a qué quieres llegar? ¿Hay suficiente información? ¿Hay información extraña? ¿Es este problema similar a algún otro que hayas resuelto antes? y dibujar una figura.

ii) Concebir un plan o elaborar un plan: analizar cómo se puede resolver el problema, es concebir la idea de un plan sin imponérselo, buscar una estrategia de resolución, con preguntas orientadoras como: ¿Puede plantear el problema en forma diferente? ¿Puede seleccionar los datos? ¿Ha empleado todos los datos? ¿Puede usar la propiedad de los números? ¿Ha hecho uso de la condición? ¿Conoce algún problema relacionado con este? ¿Conoce algún problema que tenga una incógnita similar a este? y relacionar datos con la incógnita para luego realizar un dibujo.

iii) Ejecutar el plan: llevar a cabo el plan ideado examinando los detalles y comprobando cada uno de los pasos: ¿Puede ver claramente que el paso es correcto? y ¿Puede verificar cada paso?

iv) Verificar el resultado y redactar la respuesta: mirar hacia atrás, verificar el resultado obtenido y redactar una respuesta acorde con la pregunta del problema.

Figura 2

Ejemplo de resolución de problema

\begin{tabular}{|c|c|}
\hline $\begin{array}{l}\text { Extrae los datos del dibujo y luego resuelve: } \\
\text { Diana salió de compras a Girón. Eligió una camisa, } \\
\text { un pantalón y unos zapatos } \\
\text { ¿Cuánto dinero gastó Diana en las compras? } \\
\text { ¿Cuál es la diferencia entre los zapatos y la camisa? } \\
\text { ¿Cuál es la diferencia entre los zapatos y el pantalón? }\end{array}$ & $\begin{array}{l}\text { paso 1: comprender el problema } \\
\text { camisa: } 15.500 \\
\text { pantalón: } 38.200 \\
\text { zapatos: } 42.000 \\
\text { ¿ cuánto dinero gasta Dianci an } \\
\text { las compras? } \\
\text { paso 2: elaborar un plan } \\
\text { realizar una suma } \\
\text { paso 3: ejecutar el plan } \\
\text { 15.500 } \\
38.200 \\
42.000 \\
95.700 \\
\text { Rta: Diana gasto en las compras } \\
\$ 95.700 \text { P }\end{array}$ \\
\hline
\end{tabular}

Fuente: estudiante \# 2 en la actividad 3.

La figura 2 corresponde a la actividad 3, diseñada para que los estudiantes extrajeran datos de un dibujo, que complementó el enunciado del problema, para luego ser resuelto mediante el método de Pólya. En este caso, el estudiante identificó claramente que, para comprender un problema, se debe tener claridad sobre cuáles son los datos y la variable o variables para resolverlo; por tanto, se relaciona con la categoría de análisis (C.3 Impacto) y la subcategoría de análisis (C.3 Impacto1).

Actividad de finalización: diseñada para que los estudiantes crearan y contextualizaran los nuevos conocimientos con los conocimientos previos, y los tuviesen en cuenta en la resolución de problemas del diario vivir. Este trabajo consistió en elaborar costos de producción y comercialización de un producto en el recreo del colegio, y se desarrolló por equipos. La figura 3, muestra la producción de uno de los equipos, en la cual se identificaron la categoría de análisis -C.3 Impacto- y la subcategoría de análisis -C.3 Impacto1-. 
Figura 3

Ejemplo de resolución de problemas contextualizados, desarrollados en equipo

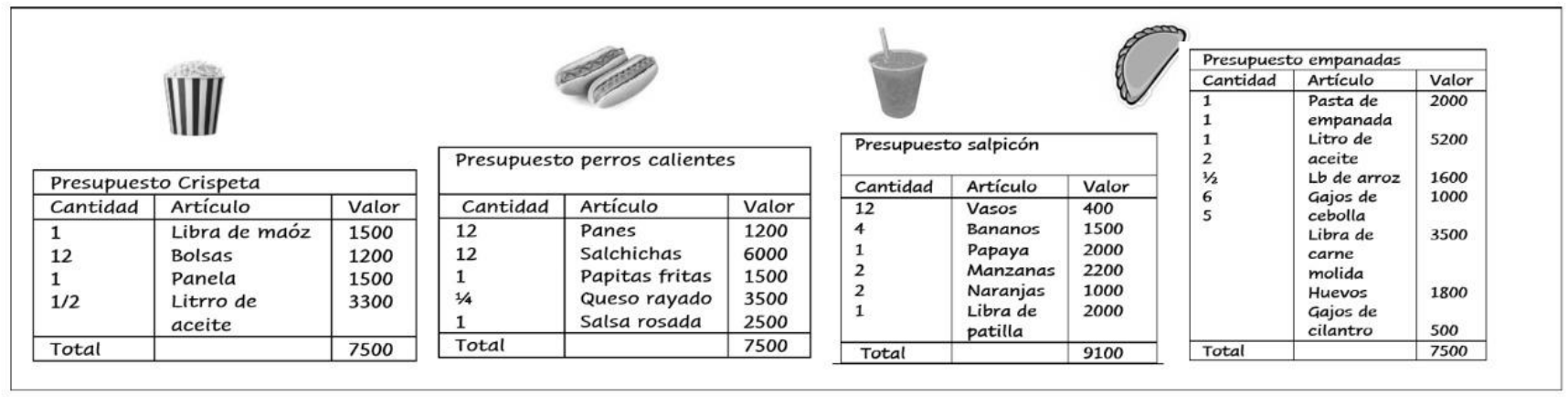

Fuente: trabajo realizado por equipos.

Prueba final: con el desarrollo de la prueba final se pudieron analizar los conocimientos adquiridos y el desarrollo de las habilidades alcanzadas por los estudiantes durante la implementación de la unidad didáctica. Se detectaron dificultades que emergieron de las categorías de análisis y que dan origen a un nuevo proceso de enseñanza y aprendizaje. Se pudo observar que los estudiantes resolvieron paso a paso los diferentes problemas. Sin embargo, persiste dificultad en algunos de ellos para resolver problemas que involucran el algoritmo de la división, así como el problema que requiere la aplicación de los algoritmos de la adición y sustracción. Además, al redactar un problema, los estudiantes acudieron a problemas sencillos del diario vivir.

También se determinó el nivel de desempeño de los estudiantes del grado cuarto al resolver problemas matemáticos, con la escala de valoración que muestra el cuadro 3.

Cuadro 3

Nivel de desempeño de los

estudiantes en la prueba final

\begin{tabular}{|c|c|c|}
\hline Problemas & Valoración & \# de estudiantes \\
\hline $0-4$ & Bajo & 1 \\
$5-6$ & Básico & 4 \\
$7-8$ & Alto & 19 \\
\hline
\end{tabular}

Fuente: elaboración propia

Como resultado, se identificó que $4 \%$ de los estudiantes se ubicó en el nivel bajo; $17 \%$ tuvo un nivel básico y $79 \%$ alcanzó el nivel alto, reflejando así una mejora en su proceso de aprendizaje de resolución de problemas matemáticos, dado el mayor número de soluciones realizadas eficientemente.

La estrategia didáctica en resolución de problemas, aquí descrita, permitió al docente contextualizar los saberes matemáticos de los estudiantes. Mediante la aplicación de actividades, el docente pudo observar el avance de los estudiantes, teniendo en cuenta de manera objetiva la aplicación de saberes y la implementación de metodologías, basadas en conjeturas e hipótesis, donde los discentes encontraron estrategias de solución y resultados verificables, lo que constituyó la construcción de nuevo conocimiento.

\section{Conclusiones}

La implementación de la unidad didáctica para fortalecer la competencia matemática resolución de problemas en los estudiantes del grado cuarto, siguiendo el método de Pólya e integrando operaciones básicas en contexto, fue de gran importancia ya que permitió a los estudiantes y docentes tener control del progreso, a medida que desarrollaron cada una de las actividades planeadas. 
Las pruebas mostraron como solo el $29 \%$ de los estudiantes aprobaron la prueba diagnóstica, mientras que el $96 \%$, es decir 23, aprobaron la prueba final, mejorando así su nivel de desempeño y cambiando en ellos, además, su actitud hacia el área de matemáticas, donde cobra sentido el ser, el saber y saber hacer.

Se fortaleció la competencia resolución de problemas, utilizando como pretexto las operaciones básicas y el método heurístico de Pólya. Esto contribuyó de forma efectiva en la disposición de los estudiantes para enfrentarse a diferentes problemas matemáticos, ejemplo de ello se puede observar en la producción del estudiante \#2, quien sigue el paso a paso del método de Pólya para resolver el problema.

Los resultados sugieren que los docentes de matemáticas deberían gestionar su clase mediante el diseño de unidades didácticas conformadas por actividades lúdicas, recreativas e innovadoras, donde se evidencie el gusto por enseñar y que incluyan el método de Pólya; estos hallazgos coinciden con los de Hoong et al. (2011). Además, los diseños instruccionales deberían integrar los conocimientos previos a los nuevos saberes, para que se aprenda haciendo. En este sentido, se debe permitir a los estudiantes: reflexionar, experimentar, comunicar y tomar decisiones en la construcción de diferentes procesos de resolución de problemas en las diversas áreas del conocimiento, en particular en el área de matemáticas; aspecto que de manera similar sugiere Jiménez-Espinoza (2019).

\section{Referencias bibliográficas}

Camero, Y., Martínez, L., \& Pérez, V. (2016). El desarrollo de la Matemática y su relación con la tecnología y la sociedad. Caso típico. Revista Universidad y Sociedad, 8(1), 97-105.

Godino, J. D., Batanero, C. \& Font, V. (2003). Fundamentos de la enseñanza y el aprendizaje para maestros. Recuperado de: http://www.ugr.es/local/jgodino/edumatmaestros

García-Arretio, L. (2009). Unidades didácticas 1. Editorial BENED. Recuperado de: http://espacio.uned.es/fez/eserv/bibliuned:23118/unidades_didacticas.pdf

García-Arretio, L. (2014). La guía Didáctica. Contextos Universitarios Mediados, 14(5), 1-8.

Gómez, P., \& Velasco, C. (2017). Complejidad y coherencia de los documentos curriculares colombianos. Revista Colombiana de Educación, 73, 261-281.

Hoong, Y., Guan, E., Lam, T., Seng, K., \& Dindyal, J. (2011). Reviving Pólya's “Look Back” in a Singapore school. Journal of Mathematical Behavior, 30, 181- 193.

Instituto Colombiano para la Evaluación de la Educación, ICFES. (2018). Guía de uso e interpretación de resultados. Reporte de estudiantes saber $3^{\circ}, 5^{\circ}$ y $9^{\circ}$. Recuperado de:

https://www.icfes.gov.co/documents/20143/1325537/Guia\%20de\%20interpretacion\%20y\%20uso\%20de \%20resultados\%20de\%20las\%20pruebas\%20saber\%20359\%20-\%20entidades-territoriales.pdf

Jiménez-Espinosa, A. (2019). La dinámica de la clase de matemáticas mediada por la comunicación. Revista de Investigación, Desarrollo e Innovación, 10 (1), 121-134. Recuperado de:

https://doi.org/10.19053/20278306.v10.n1.2019.10016

Li, L., Zhoua, X., Huanga, J., Tuc, D., Gaod, X., Yanga, Z., \& Lia, M. (2020). Assessing kindergarteners' mathematics problem solving: The development of a cognitive diagnostic test. Studies in Educational Evaluation, 66, 1-10. 
López-Leyton, C., Aldana, E. \& Erazo, J. (2019). El papel de la resolución de problemas para la enseñanza del cálculo integral: un estudio de caso. Revista Espacios, 40(17), 12. Recuperado de:

https://www.revistaespacios.com/a19v40n17/a19v40n17p12.pdf

Marín, J. D. (2018). Investigar en educación y pedagogía: sus fundamentos epistemológicos y metodológicos. Bogotá, Colombia: Editorial Magisterio.

Mazzilli, D. M., Hernández, L. E., \& De La Hoz, S. I. (2016). Procedimiento para desarrollar la competencia matemática resolución de problemas. Escenarios, 14 (2), 103-119. Recuperado de: http://dx.doi.org/10.15665/esc.v14i2.935

Ministerio de Educación Nacional de Colombia, MEN. (1998). Lineamientos Curriculares Matemáticas. Santa Fe de Bogotá, Colombia: Delfín.

Ministerio de Educación Nacional de Colombia, MEN. (2006). Estándares Básicos de Competencias en Lenguaje, Matemáticas, Ciencias y Ciudadanas. Santa Fe de Bogotá, Colombia: Ministerio de Educación Nacional.

Ministerio de Educación Nacional de Colombia, MEN. (2016). Derechos Básicos de Aprendizaje V2. Recuperado de: http://aprende.colombiaaprende.edu.co/sites/default/files/naspublic/DBA_Matem \%C3 \%A1ticas.pdf

Organización para la Cooperación y Desarrollo Económicos, OCDE. (2017). Marco de Evaluación y de Análisis de PISA para el Desarrollo: Lectura, matemáticas y ciencias. Versión preliminar, Paris, Francia: OECD Publishing.

Phonapichat, P., Wongwanich, S., \& Sujiva, S. (2014). An analysis of elementary school students' difficulties in mathematical problem solving. Procedia - Social and Behavioral Sciences, 116, 3169-3174.

Pólya, G. (1965). Como plantear y resolver problemas. Ciudad de México, México: Editorial Trillas.

Sánchez, J. C., \& Fernández, J. A. (2003). La enseñanza de la matemática. Fundamento teórico y bases psicopedagógicas. Madrid, España: CCS Alcalá.

Sandín, E, M. (2003). Investigación cualitativa en educación. Fundamentos y tradiciones. Madrid, España: McGraw-Hill.

Santos-Trigo, L. (2008). La resolución de problemas matemáticos: avances y perspectivas en la construcción de una agenda de investigación y práctica. Investigación en Educación Matemática XII. Badajoz, España.

Valbuena, S., Muñiz, L. \& Berrio, J. (2020). El rol del docente en la argumentación matemática de estudiantes para la resolución de problemas. Revista Espacios, 41(9), 15. Recuperado de:

https://www.revistaespacios.com/a20v41n09/a20v41n09p15.pdf

Villalobos, F. X. (2008). Resolución de problemas matemáticos: un cambio epistemológico con resultados metodológicos. Revista Iberoamericana sobre calidad, eficiencia y cambio en educación, 6 (3), 36-58.

Esta obra está bajo una Licencia Creative Commons Attribución-NoCommercial 4.0 International

(cc) EY-NC 